\title{
Investigation and Therapeutic Management of Hymenolepiosis in Wistar Rats from Chhattisgarh
}

\author{
Nidhi Rawat ${ }^{1 *}$, Dhananjay Kumar Jolhe ${ }^{2}$, Sadanand Devidas Hirpurkar ${ }^{1}$, Savita Bisen ${ }^{3}$, \\ Shailesh Kumar Patel ${ }^{2}$, Chandrahas Sannat ${ }^{1}$ and Seema Mandavi ${ }^{4}$ \\ ${ }^{1}$ Department of Veterinary Microbiology, College of Veterinary Science and Animal Husbandry, Anjora, Durg, \\ Chhattisgarh, INDIA \\ ${ }^{2}$ Department of Veterinary Pathology, College of Veterinary Science and Animal Husbandry, Anjora, Durg, Chhattisgarh, INDIA \\ ${ }^{3}$ Department of Veterinary Parasitology, College of Veterinary Science and Animal Husbandry, Anjora, Durg, \\ Chhattisgarh, INDIA \\ ${ }^{4}$ Department of Veterinary Anatomy, College of Veterinary Science and Animal Husbandry, Anjora, Durg, Chhattisgarh, INDIA
}

"Corresponding author: N Rawat; E-mail:dr_nidhirawat@yahoo.com

Received: 20 May, 2020

Revised: 14 July, 2020

Accepted: 16 July, 2020

\begin{abstract}
The present investigation was aimed to know the cause of sudden mortality among Wistar rats in a rat colony. The dead rats $(n=20)$ were subjected to necropsy examination. Intestinal lumens were examined to know the presence of parasites or parasitic body parts. Intestinal faecal contents were collected for identification of the parasitic eggs. Intestinal tissues were collected for histopathological examination. Necropsy revealed the presence of cestodes in the intestinal lumen. The intestines were found to be haemorrhagic and filled with serous and blood tinged contents. The cestode worms were identified as Hymenolepis diminuta based on gross morphological examination of the adult worms and microscopic examination of the eggs. Histopathology revealed necrosis of enterocytes, blunting of villi, degeneration and necrosis of villous and crypt epithelium. The diseased rats were observed for clinical signs and symptoms. Clinically, the rats showed inappetence, dullness, depression, loss of body weight, rough hair coat and a typical hunchback posture. The affected rats were treated by oral administration of Praziquantel, Pyrantel Pamoate and Febantel combination (kiwof plus tablet, Savavet) at a dose rate of $6 \mathrm{mg} / \mathrm{kg}$ body weight. The rats were cured after anthelmintic treatment. The present study highlighted the occurrence of hymenolepiosis that caused high mortality among Wistar rats. This study emphasizes the necessity of rapid and specific disease diagnosis and treatment to control the mortality among affected rats and to prevent and control the further spread of parasitic infection among susceptible rats and in the personnel in close surroundings of the infected rats.
\end{abstract}

\section{HIGHLIGHTS}

(0 Histopathology of intestine revealed necrosis of enterocytes, blunting of villi, degeneration and necrosis of villous and crypt epithelium.

(0 Gross and microscopic examination of the cestode worms indicated Hymenolepis diminuta.

(- Affected rats were cured after oral administration of praziquantel, pyrantel pamoate and febantel.

Keywords: Hymenolepis diminuta, mortality, therapeutic management, Wistar rat, zoonoses

Hymenolepis diminuta, commonly known as "rat tapeworm" causes hymenolepiosis in rodents including rat (Yang et al., 2017; Sood et al., 2018). The disease has been reported from most of the Asian countries including India (Goswami et al., 2009; Goswami et al., 2011; Singh and Arya, 2015), China (Yang et al., 2017), Pakistan
(Ahmad et al., 2014), Indonesia (Priyanto et al., 2014),

How to cite this article: Rawat, N., Jolhe, D.K., Hirpurkar, S.D. Bisen, S., Patel, S.K., Sannat, C. and Mandavi, S. (2020). Investigation and therapeutic management of hymenolepiosis in Wistar rats from Chhattisgarh. J. Anim. Res., 10(4): 609-614.

Source of Support: None; Conflict of Interest: None 
Iran (Pakdel et al., 2013), Malaysia (Zain et al., 2012), etc. $H$. diminuta in rats is transmitted by arthropod vectors. The affected rats show inappetence, dullness, weakness, loss of body weight, pale mucous membrane, rough haircoat and mortality (Singh and Arya, 2015). Eggs of $H$. diminuta are released in faeces of the infected host. Mature eggs of the tapeworm are ingested by an intermediate host (various arthropod adults or larvae). The oncospheres are released from the eggs and penetrate the intestinal wall of the arthropod and develop into cysticercoid larvae. The definitive host acquires infection by ingestion of the infected arthropods. Occasionally, humans are infected by accidental ingestion of the infected arthropods, and thus $H$. diminuta has a zoonotic significance (Watwe and Dardi, 2008; Karuna and Khadanga, 2013; Saha et al., 2017). Rodents such as rats, mice, guinea pigs, hamsters etc are commonly used to perform the experimental studies. Therefore, these experimental animals must be healthy and free from pathogens including parasites as their infection can affect the outcome of the experimental studies. Therapeutic management of hymenolepiosis depends upon rapid and specific diagnosis of this disease. Therefore, the present investigation was carried out to know the exact cause of the disease and to check the mortality among diseased rats by using suitable and effective anthelmintic treatment.

\section{MATERIALS AND METHODS}

Overview of the study area, animal housing, and management

The study area lies between $20^{\circ} 54^{\prime}$ and $21^{\circ} 32^{\prime}$ north latitude and $81^{\circ} 10^{\prime}$ and $81^{\circ} 36^{\prime}$ east longitude. The area is about 317 meters high from the sea level. It is hot and humid in the summer and monsoon seasons. A total of 76 Wistar rats were maintained at a small animal house facility at the College of Veterinary Science and Animal Husbandry, Anjora, Durg, Chhattisgarh. The rats were reared by intensive management system where these animals were provided with formulated concentrate feed (pelleted) and purified water (by bottles). The rats were maintained in polypropylene cages (with dried husk litter material) kept in a cemented house enclosure with a controlled ambient temperature of $25^{\circ} \mathrm{C}$ using air conditioners.

\section{Disease overview and clinical examination}

At the beginning of January 2019, a high morbidity with mortality was recorded among Wistar rats. The diseased rats showed loss of appetite, dullness, depression, loss of body weight, rough haircoat, and a typical hunchback posture (Fig. 1). Twenty (26.3\%) out of 76 rats died during a period of one week. The diseased rats were observed for clinical signs and symptoms of hymenolepiosis. The rats were also observed for any change in their behaviour. Faecal samples from the affected rats were collected and examined for the presence of parasitic eggs.

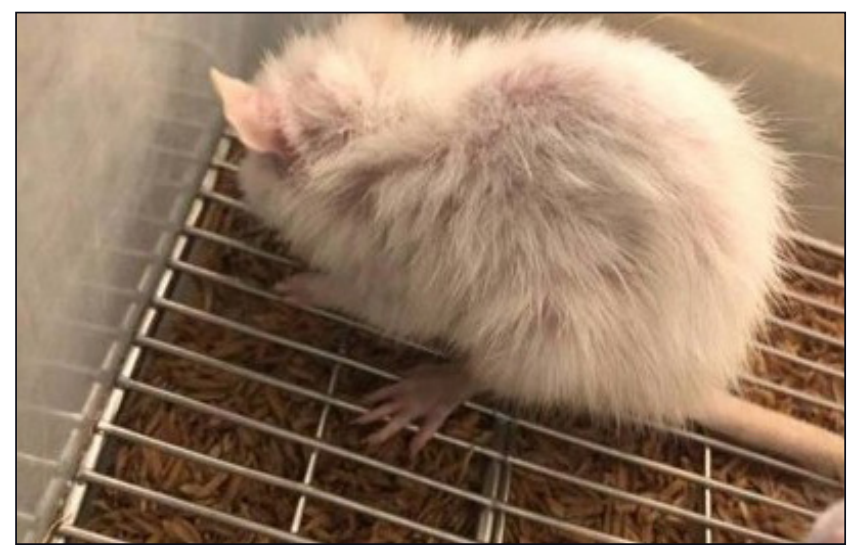

Fig. 1: Rough haircoat and typical hunchback posture of affected rat

\section{Necropsy examination and sample collection}

A detailed examination necropsy of 20 fresh dead Wistar rats was performed to know the pathological changes. Intestinal parts were collected for detailed examination of the parasites. Moreover, the small intestinal tissues were collected in 10\% neutral buffered formalin for histopathological examination. Simultaneously, the faecal content from the intestinal lumens was collected in sterile plastic specimen containers and subjected to microscopic examination for detection and identification of parasitic eggs.

\section{Identification of parasites and their eggs}

The parasite worms were removed carefully from the intestinal lumen, cleared in sterile phosphate buffered saline and examined for the presence of adult or larval stages of cestodes under a light microscope. Faecal 
samples were subjected to wet smear examination under microscope to identify the parasitic eggs.

\section{Histopathological examination}

The intestinal tissues were processed for histopathological examination using routine paraffin-embedded technique. About $5 \mu \mathrm{m}$ thick sections were cut and stained with haematoxylin and eosin ( $\mathrm{H} \& \mathrm{E})$ stain as per Luna (Luna, 1968).

\section{Treatment of the diseased rats}

Treatment of the affected rats was done by single oral administration of Praziquantel, Pyrantel Pamoate and Febantel combination (kiwof plus tablet, Savavet) at the dose rate of $6 \mathrm{mg}$ per $\mathrm{kg}$ of body weight. The medicine was dissolved in purified water and administered to the individual rats using the droppers. The anthelmintic therapy was repeated after 15 days. The efficacy of anthelmintic drug was assessed by successful expulsion of the cestode or its proglottids after the therapy and by ascertaining the absence of characteristic parasitic eggs in the faecal samples.

\section{RESULTS AND DISCUSSION}

The post-mortem examination of the rat carcasses showed haemorrhages in the intestines. The intestines were filled with serous and blood tinged contents. Intestinal lumen was filled with large number of parasitic worms (tapeworms) (Fig. 2-3). There was thinning of the intestinal wall, and the tapeworms could be seen from the serosal surface of the intestines. Other visceral organs except lungs did not reveal specific pathological alterations. The lungs were seen congested and haemorrhagic among few rats.

The parasites measured $60-80 \mathrm{~mm}$ in length and $2-4 \mathrm{~mm}$ in width. Segments of the tapeworms with serrated borders were seen during microscopic examination (Fig. 4). In a few rats, the tapeworm scolices were found to be attached to the intestinal mucosa. The parasitic scolices had four suckers without the rostellar hooks.

Microscopic examination of the faeces and intestinal contents showed the presence of eggs specific to $H$. diminuta. The eggs were yellowish-brown in colour and round to oval shaped and measured about 70-80 $\mu \mathrm{m}$ (length) $\times 60-70 \mu \mathrm{m}$ (width) by micrometry. Microscopic examination revealed eggs having hexacanth embryo with three pairs of hooks and the absence of polar filaments (Fig. 5).

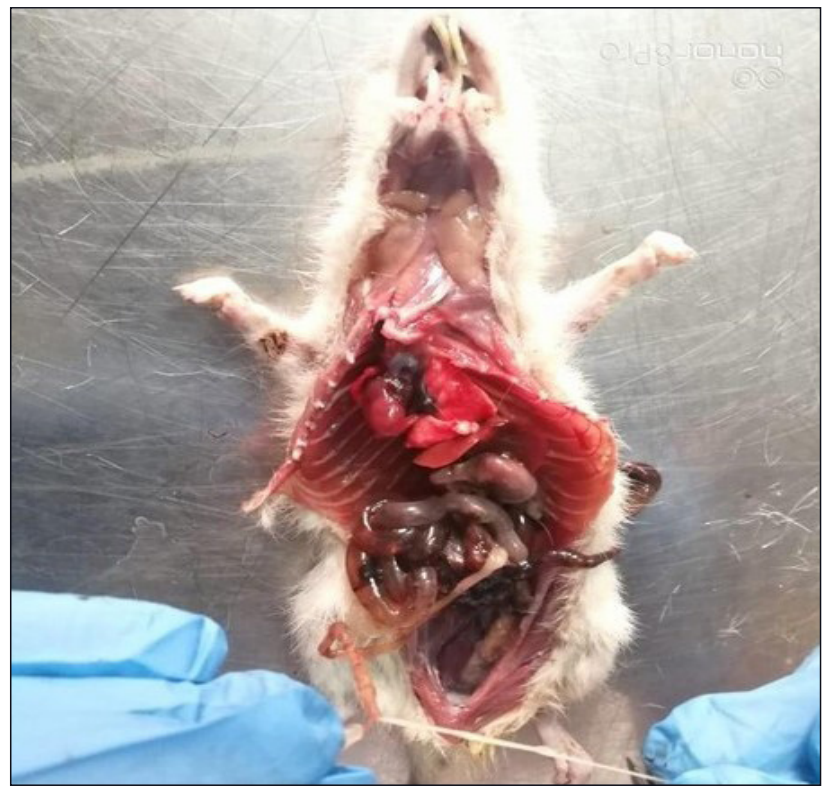

Fig. 2: Haemorrhagic and occluded intestines due to $H$. diminuta

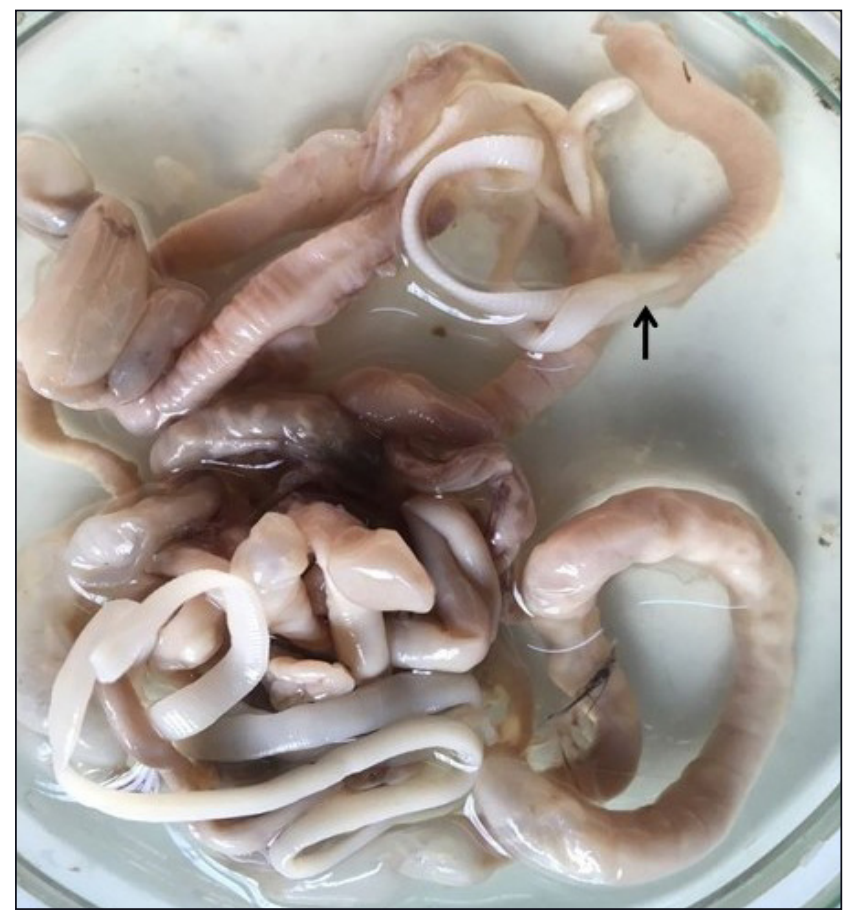

Fig. 3: Rat intestines occluded by tapeworms 


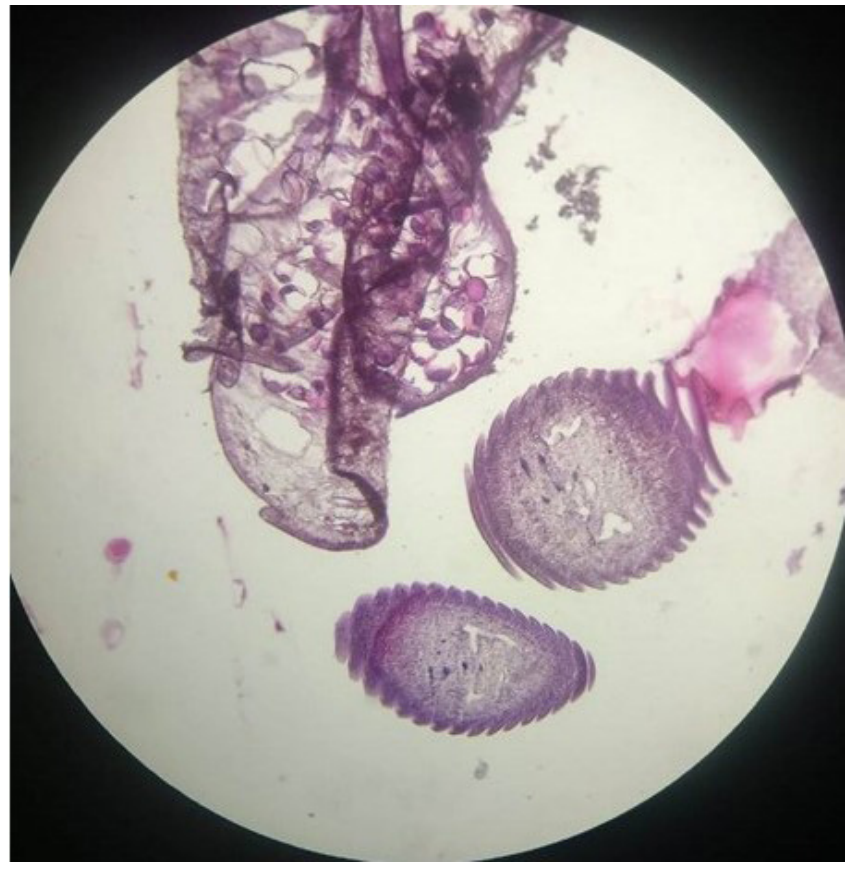

Fig. 4: Parts of tapeworm with serrated border found in rat intestine

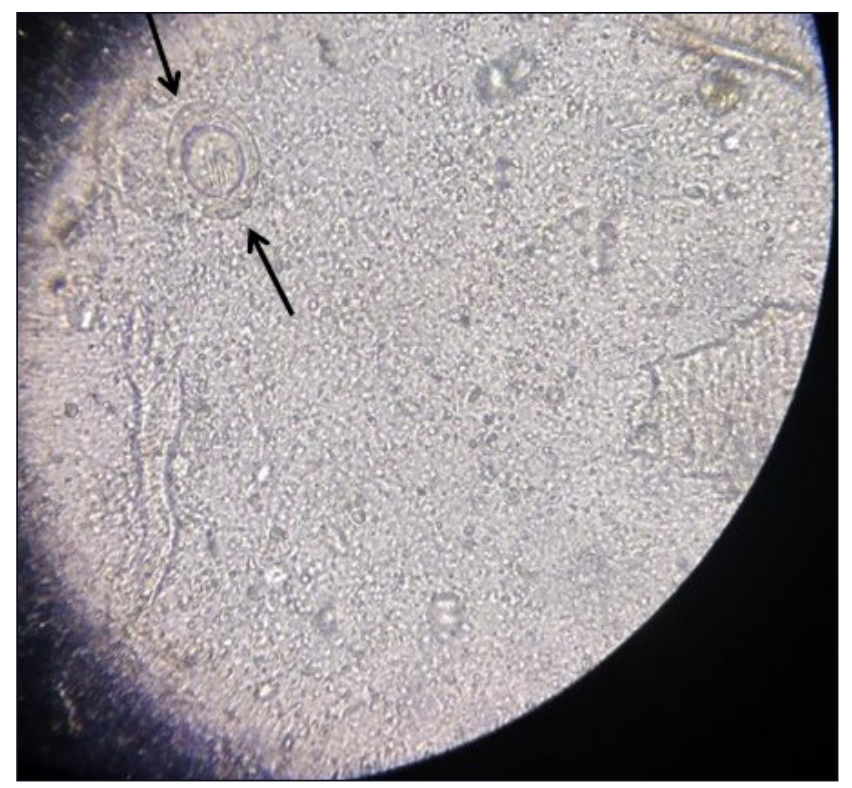

Fig. 5: Round-to-oval shaped egg of $H$. diminuta having oncosphere and three pairs of hooks

Disease diagnosis could be done to identify the causative parasitic species by specific morphological features and by the identification of specific tapeworm eggs (Nkouawa et al., 2016). Disease characteristics and mortality rate with more or less similarity have been reported earlier (Pakdel et al., 2013; Singh and Arya, 2015; Yang et al., 2017). However, Ranjbar et al. (2017) reported a higher (50\%) prevalence of $H$. diminuta among the captured rodents in South-Western Iran. The findings of the present study could be correlated with the fact that a high worm burden disturbs the normal physiology of the infected host (Baker, 2007). However, the mild infections of $H$. diminuta have been observed as non-pathogenic to the rats (Insler and Roberts, 1976). Clinically affected rats showed a pale-yellowish discolouration of the mucous membranes indicating anaemia (Goswami et al., 2011). Emaciation along with catarrhal haemorrhagic enteritis was seen as the common finding among the dead rats. The intestinal impaction due to heavy burden of the cestode worms might have led to the increased intestinal permeability with varying degree of catarrhal enteritis and enterocolitis. The host body defense mechanism could lower the $H$. diminuta load (Andreassen and Hopkins, 1980) that might be a reason of the low worm burden among other rats of the same colony. Histopathology of small intestinal tissues showed necrosis of enterocytes, blunting of villi, degeneration and necrosis of villous and crypt epithelium and infiltration of eosinophils and mononuclear cells in the mucosa (Fig. 6). There was an excessive mucous production by intestinal mucosal cells. The histopathological findings could be supported with the results of the previous studies on $H$. diminuta infections in rats (Goswami et al., 2011; KosikBogacka and Kolasa, 2012).

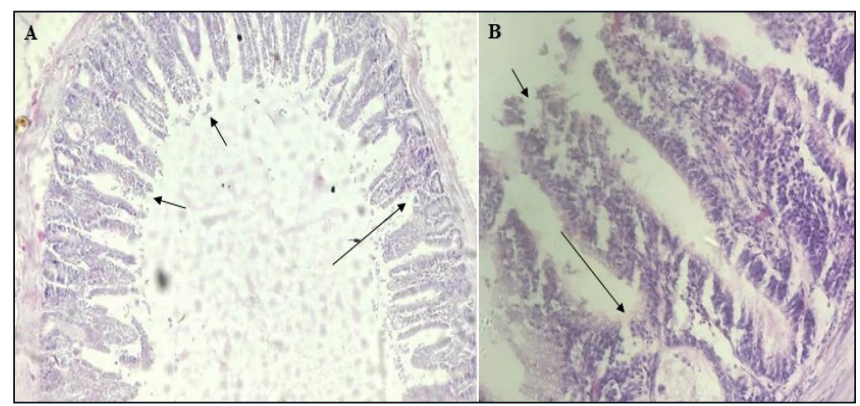

Fig. 6 A-B: Histopathology of intestine showing blunting of villi, degeneartion and necrosis of villous (small arrow) and crypt epithelium (large arrow) with inflammatory cells. A: $40 \times$ and $\mathrm{B}$ : $100 \times$ magnification

Absence of cestode eggs in faeces and successful expulsion of the tapeworms and/or its proglottids from the 
rat intestine indicated the therapeutic efficacy of the used anthelminthic drugs. All clinically affected rats were cured after anthelmintic therapy. The results of anthelmintic therapy against $H$. diminuta using a single dose of Praziquantel were corroborated by Fan and Ito (1995) and Garg et al. (2007). However the present findings are contrary to the results of Tena et al. (1998) who reported a single dose $(10 \mathrm{mg} / \mathrm{kg}$ of body weight $)$ of Praziquantel to be ineffective against $H$. diminuta infection in human but the parasite was eradicated after three treatment cycles using the same drug at dosages of $25 \mathrm{mg} / \mathrm{kg}$ /day for 5 days.

The stored litter and feed in the small animal house unit were discarded and replaced by a new lot. Moreover, all the cages, water bottles, feeding pans, and the house floors were properly cleaned.

The present study highlighted an outbreak of hymenolepiosis caused by $H$. diminuta among Wistar rats maintained in an organized small animal house unit at Durg district of the Chhattisgarh state. $H$. diminuta infection was found to be the cause of death of 20 rats accounting for a high prevalence $(26.3 \%)$ of this tapeworm parasite. The parasite was specifically identified based on the morphological features of the adult worms and their eggs. Histopathological observations revealed the catarrhal, degenerative and necrotic changes in the intestinal mucosa. The clinically affected rats were cured after a single oral administration of Praziquantel. Since the rodents can harbour cestodal worms with sub-clinical infections, the personnel working in close proximity of the rodents may be at risk of infections that could be acquired unknowingly through infected arthropods. Therefore, a specific diagnosis and treatment of hymenolepiosis is helpful to control the mortality among affected rats and to restrict the spread of causative parasites to the susceptible rats or to the human population.

\section{ACKNOWLEDGEMENTS}

The authors are thankful to the Dean of College of Veterinary Science and Animal Husbandry, Anjora, Durg, Chhattisgarh for providing the necessary facilities to conduct the present research work.

\section{REFERENCES}

Ahmad, M.S., Maqbool, A., Anjum, A.A., Ahmad, N., Khan,
M.R., Sultana, R. and Ali, M.A. 2014. Occurrence of Hymenolepis diminuta in rats and mice captured from urban localities of Lahore. J. Anim. Plant Sci., 24 (2): 392-396.

Andreassen, J. and Hopkins, C.A. 1980. Immunologically mediated rejection of Hymenolepis diminuta by its normal host, the rat. J. Parasitol., 66(60): 898-903.

Baker, D.G. 2007. Parasites of rats and mice. Flynn's parasites of laboratory animals. $2^{\text {nd }}$ Ed., Blackwell publishing, Ames.

Fan, P. and Ito, A. 1995. The minimum effective dose of praziquantel in treatment of Hymenolepis diminuta in rats. $J$. Helminthol., 69(1): 91-92.

Garg, R., Vatsya, S., Yadav, C.L., Banerjee, P.S. and Hore, S.K. 2007. Treatment of Hymenolepis sp. infection in rats using different anthelminthics. J. Vet. Parasitol., 21(1): 75-76.

Goswami, R., Singh, S.M., Kataria, M. and Somvanshi, R. 2011. Clinicopathological studies on spontaneous Hymenolepis diminuta infection in wild and laboratory rats. Braz. J. Vet. Pathol., 4(2): 103-111.

Goswami, R., Somvanshi, R., Singh, S.M. and Sarman, S. 2009. A preliminary survey on incidence of helminthic and protozoal diseases in rats. Indian J. Vet. Pathol., 33(1): 90-92.

Insler, G.D. and Roberts, L.S. 1976. Hymenolepis diminuta: lack of pathogenecity in the healthy rat host. Exp. Parasitol., 39(3): 351-357.

Karuna, T. and Khadanga, S. 2013. A case of Hymenolepis diminuta in a young male from Odisha. Trop. Parasitol., 3(2): 145-147.

Kosik-Bogacka, D.I. and Kolasa, A. 2012. Histopathological changes in small and large intestines during hymenolepidosis in rats. Folia Biol., 60(3-4): 195-198.

Luna, L.G. 1968. Manual of histologic staining methods of the armed forces, Institute of pathology. $3^{\text {rd }}$ Ed. Mc Graw Hill Book Company, New York, USA.

Mohd Zain, S.N., Behnke, J.M. and Lewis, J.W. 2012. Helminth communities from two urban rat populations in Kuala Lumpur, Malaysia. Parasit. Vectors., 5: 47.

Nkouawa, A., Haukisalmi, V., Li, T., Nakao, M., Lavikainen, A., Chen, X., Henttonen, H. and Ito, A. 2016. Cryptic diversity in hymenolepidid tapeworms infecting humans. Parasitol. Int., 65(2): 83-86

Pakdel, N., Naem, S., Rezaei, F. and Chalehchaleh, A.A. 2013. A survey on helminthic infection in mice (Mus musculus) and rats (Rattus norvegicus and Rattus rattus) in Kermanshah, Iran. Vet. Res. Forum., 4(2): 105-109.

Priyanto, D., Rahmawati and Ningsih, D.P. 2014. Identification of endoparasites in rats of various habitats. Health Sci. Indones., 5 (1): 49-53. 
Ranjbar, M.J., Sarkari, B., Mowlavi, G.R., Seifollahi, Z., Moshfe, A., Abdolahi Khabisi, S. and Mobedi, I. 2017. Helminth infections of rodents and their zoonotic importance in BoyerAhmad District, Southwestern Iran. Iran. J. Parasitol., 12(4): 572-579.

Saha, R., Kaushik, S., Gupta, K. and Das, S. 2017. Human Infection with Hymenolepis diminuta: first case report from North India. J. Gastrointest. Infect., 7(1): 36-37.

Singh, Y.D. and Arya, R.S. 2015. Clinico-pathology, diagnosis and management of Cysticercus fasciolaris and Hymenolepis diminuta co-infection in wistar rats, Vet. World, 8(1): 116120.

Sood, S., Yadav, A., Katoch, R., Ganai, R.A. and Imtiaz, S. 2018. Hymenolepis nana infection in laboratory rats: A case report. J. Entomol. Zool. Stud., 6(4): 1202-1204.
Tena, D., Perez Simon, M., Gimeno, C., Perez Pomata, M.T., Illescas, S. Amondarain, I., Gonzalez, A., Dominguez, J. and Bisquert, J. 1998. Human infection with Hymenolepis diminuta: case report from Spain. J. Clin. Microbiol., 36(8): 2375-2376

Watwe, S. and Dardi, C.K. 2008. Hymenolepis diminuta in a child from rural area. Indian J. Pathol. Microbiol., 51(1): 149-150.

Yang, D., Zhao, W., Zhang, Y. and Liu, A. 2017. Prevalence of Hymenolepis nana and $H$. diminuta from Brown Rats (Rattus norvegicus) in Heilongjiang Province, China. Korean $J$. Parasitol., 55(3): 351-355. 